\title{
Relações Internacionais: objeto e fronteira das ciências sociais?
}

\author{
International Relations: object and frontier of the Social Sciences?
}

\section{Maria Caramez Carlotto}

O objetivo geral do presente dossiê, como o título sugere - "Relações Internacionais: objeto ou fronteira das ciências sociais?” -, é explorar as tensões teóricas e metodológicas no tratamento do “internacional” entre as Relações Internacionais, como disciplina, os ensaios interdisciplinares que apontam para a consolidação de novas áreas, como a Economia Política Internacional, e outras ciências sociais consolidadas, em especial a Sociologia. A motivação de endereçar este problema específico em um dossiê no Brasil é dupla.

Em primeiro lugar, é preciso reconhecer que, nos últimos anos, tem crescido o diálogo entre as ciências sociais, em particular a Sociologia, e outras tradições disciplinares e interdisciplinares que pensam o internacional, como as Relações Internacionais e a Economia Política Internacional. Expressão importante disso é o crescimento das publicações que tematizam justamente esse diálogo. Sem sermos exaustivos, podemos citar nessa linha, por exemplo, o volume organizado por Mathias Albert e Lena Hilkermeier, na The New International Relations Series da Routdledge, intitulado Observing International Relations: Niklas Luhmann and World Politics (2003). Ou ainda, na mesma série, a coletânea de Rebeca Adler-Nissen, Bourdieu in International Relations: Rethinking Key Concepts in International Relations (2013). Adler-Nissen, que é autora, ainda, do artigo On a Field Trip with Bourdieu (2011), caminha na mesma direção dos influentes trabalhos de Didier Bigo como Bourdieu and International Relations: Power of Practices and Practices of Power (2011) e Security, IR [International Relations] and Anthropology: Encounters, Misunderstanding and Possible Collaborations (2014).

Ainda nessa fronteira da sociologia contemporânea, encontram-se os esforços de Bruno Latour para se aproximar do campo das Relações Internacionais, tal como explícito em artigos como Some Advantages of the Notion of "Critical Zone” for Geopolitics (2014) e 'Onus Orbis Terrarum': About a Possible Shift in the Definition of Sovereignty (2016), ou ainda, na entrevista Bruno Latour

a Professora da Universidade Federal do ABC e membro do grupo de pesquisa Filosofia, História e Sociologia da Ciência e da Tecnologia, sediado no Instituto de Estudos Avançados da USP. 
encounters International Relations, publicada em 2017 no Millenium: Journal of International Studies. Também vale lembrar o recém lançado Foucault and the Modern International: Silences and Legacies for the Study of World Politics (2017). Por fim, podemos citar todo um conjunto de sociólogos que passaram a orientar seus trabalhos justamente para o tema do internacional, em diálogo explícito ou implícito com o campo das Relações Internacionais (CAmpbell, 2009; Dezalay \& Garth, 1998, 2002 e 2006; Gullhot, 2005; Fourcade, 2006; Martin, MetzGer E Pierre, 2006; entre outros).

Mas, para além do florescimento desse diálogo teórico-metodológico em âmbito internacional, o presente dossiê encontra sua motivação na crescente consolidação da disciplina de Relações Internacionais no Brasil, que ainda requer um balanço mais aprofundado quanto aos seus impactos para o campo nacional das Ciências Sociais como um todo.

Apesar de parecer, hoje, uma área bastante consolidada, as Relações Internacionais, como disciplina acadêmica, só ganharam densidade no país nas últimas duas décadas. Um marco fundamental desse processo foi o aumento significativo do número de cursos de graduação no tema a partir dos anos 1990. De fato, com exceção do curso da UnB, criado em 1974, todos os demais cursos de graduação em Relações Internacionais atualmente existentes no país só surgiram a partir da segunda metade dos anos 1990. Em 2016, segundo dados oficiais do Ministério da Educação, eram 95 cursos de Relações Internacionais em funcionamento no país, o que mostra um crescimento expressivo em duas décadas.

No âmbito da pós-graduação, a evolução é ainda mais recente. Depois da criação dos programas de pós-graduação da UnB e da PUC-Rio na década de 1980, as pós-graduações em Relações Internacionais só voltaram a surgir pós anos 2000. Atualmente, existem 11 programas de pós-graduação da área no país, o que significa que a subárea de Relações Internacionais, hoje vinculada ao comitê de Ciência Política da CAPES, cresce a uma velocidade maior do que a média da pós-graduação nacional. Esse desenvolvimento institucional e acadêmico ganha novos contornos e desdobramentos com a recente criação de cursos interdisciplinares - portanto, com mais autonomia em relação à Ciência Política do que as Relações Internacionais - também voltados à pesquisa sobre "o internacional". São exemplos desse desdobramento o Programa de Pós-Graduação em Economia Política Mundial, da UFRJ, e o Programa de Pós-Graduação em Economia Política Mundial da UFABC, que começa a funcionar em 2018 com mestrado e doutorado e vinculado ao comitê interdisciplinar da CAPES. 
O desenvolvimento da área de Relações Internacionais no Brasil teve, ainda, dois marcos importantes. Primeiro, a criação da Associação Brasileira de Relações Internacionais (ABRI) em setembro de 2005, que simboliza a consolidação de uma comunidade de pesquisa com relativa autonomia. Segundo, o projeto de lei $\mathrm{n}^{\circ}$ 8.777 de novembro de 2017 "que dispõe sobre o exercício da profissão de bacharel em Relações Internacionais e autoriza a criação dos respectivos Conselho Federal e Conselhos Regionais dos profissionais em Relações Internacionais”. Apesar de ainda enfrentar resistência da própria comunidade de pesquisa na área, ${ }^{1}$ o projeto de regulamentação da profissão é um indício importante do fortalecimento das Relações Internacionais, como subárea das Ciências Sociais, no país. Pesquisas recentes apontam, ainda, que essa afirmação das Relações Internacionais, que se traduz no aumento e na afirmação da sua comunidade epistêmica, é comum também a outros países da América Latina (VILLA ET AL., 2017, p. 227).

Para contextualizar melhor essa dupla motivação do presente dossiê - qual seja, a intensificação do diálogo entre as diferentes abordagens disciplinares e interdisciplinares que se dedicam à pesquisa do internacional e o recente esforço de consolidação da disciplina de Relações Internacionais no Brasil - é preciso recuperar, em linhas gerais, a história intelectual e institucional dessa área.

As disciplinas constitutivas das Ciências Sociais modernas - em especial, a Sociologia, a Ciência Política, a Antropologia e a Economia - emergiram, como se sabe, já no quadro histórico dos Estados Nacionais e no âmbito das universidades de pesquisa, como instituições centrais do Estado-nação moderno. Embora a dimensão internacional tenha, desde sempre, integrado o escopo de preocupação dessas disciplinas, nenhuma delas tomou o internacional como objeto privilegiado de investigação. As Relações Internacionais, que têm essa pretensão, são, por sua vez, a mais tardia das disciplinas que constituem as Ciências Sociais contemporâneas.

1 O maior indício disso é o fato de que a ABRI posicionou-se contrariamente ao projeto. Interessante notar que a oposição não é exatamente ao esforço de regulamentação da profissão de internacionalista, mas ao momento em que isso está sendo proposto. Segundo a nota da associação: "Embora agradecendo a todas e todos que se envolveram na elaboração, apresentação e tramitação do referido Projeto de Lei por seu interesse pela nossa área de atividade, a ABRI entende que, pelo menos nesse momento, a referida regulamentação prejudicará o desenvolvimento da área no Brasil, ao invés de beneficiá-la. Em que pese o amplo desenvolvimento, no Brasil, da formação em Relações Internacionais, nos níveis de graduação e de pós-graduação, o campo de atividades das profissionais e dos profissionais da área ainda está em constituição. (...) A ABRI teme, assim, que, apesar das boas intenções que levaram ao Projeto de Lei $n^{0}$ 8777/2017, uma regulamentação das atividades esteja em desconexão com o atual estágio de desenvolvimento da área de Relações Internacionais nos mercados de trabalho público e privado, e acabe por limitar, ao invés de estimular, as oportunidades de atuação profissional em Relações Internacionais" (ABRI, 2017). 
A sua historiografia clássica, pensada de uma perspectiva endógena, enfatiza a importância dos embates teóricos e dos problemas científicos que levaram ao desenvolvimento intelectual da disciplina, visto, dessa perspectiva, como uma necessidade histórica ligada ao próprio progresso da Razão (FoucAult, 2010). Segundo essa visão específica, ganha destaque a inflexão supostamente antinormativa que está na origem da crítica do idealismo liberal pelo "realismo", até hoje uma das correntes hegemônicas da disciplina (VILLA ET AL., 2017). No entanto, partindo de um enquadramento teórico mais crítico-institucional, trabalhos recentes como The discipline of Western Supremacy, terceiro volume da trilogia Modes of Foreign Relations and Political Economy (2014), de Kess van der Pijl , e The Invention of Internacional Relations Theory: Realism, the Rockfeller Foundation and the 1954 Conference, organizado pelo francês Nicolas Guilhot (2011), têm enfatizado outras dimensões da história da disciplina, como o fato da sua origem se ligar diretamente aos esforços dos governos inglês, no pós-Primeira Guerra, e norte-americano, no pós-Segunda Guerra, para consolidar uma expertise acadêmica que analisasse, orientasse e legitimasse a política externa dessas potências (Guilhot, 2011; PiJl, 2014).

Kees van der Pijl, partindo de um escopo histórico mais amplo que remete à Pax Britannica do século XIX e que tem na Revolução de 1917 a sua grande inflexão, desenvolve uma abordagem bastante crítica dos pressupostos "impensados" da teoria hegemônica de Relações Internacionais. Citando explicitamente Bourdieu e sua crítica às ciências que assumem como necessidades históricas o que na verdade são arbitrários sociais, Pijl constrói toda uma crítica à naturalização, por parte dos teóricos hegemônicos de Relações Internacionais, de um modo historicamente dado de relações exteriores, marcadas por "um complexo de Estados-soberanos sob a tutela de uma autodenominada 'comunidade internacional' sediada em Londres ou Washington” (PııL, 2014, p. vi). Essa construção teórica específica - que pressupõe como "natural" a organização do sistema internacional ligado à origem do capitalismo e do imperialismo inglês e norte-americano - é o que leva Pjil a considerar tanto as vertentes hegemônicas da teoria de Relações Internacionais quanto o campo teórico que se estrutura a partir delas como uma forma de ideologia que pode ser melhor compreendida, na verdade, como a disciplina - no duplo sentido do termo - da supremacia ocidental (PJIL, 2014).

Já a coletânea organizada por Guilhot (2011) parte de um recorte histórico mais circunscrito e uma perspectiva crítica mais nuançada para, porém, chegar a conclusões muito próximas das de Pjil sobre o caráter ideológico e politicamente útil da teoria estruturante do campo de Relações Internacionais. O volume concentra-se 
na atuação da Fundação Rockfeller no pós-Segunda Guerra Mundial para reconfigurar o campo acadêmico das Ciências Sociais, a partir da consolidação de uma disciplina voltada exclusivamente à tarefa de pensar "cientificamente" o político, e, dentro dela, em particular as relações exteriores. A história institucional da Ciência Política já tinha sido trabalhada por Guilhot em The Democracy Makers (2005), que persegue algumas pistas da pesquisa do seu orientador, Yves Dezalay, sobre as dinâmicas de exportação/importação de saberes de Estado entre as academias norte e latino-americanas a partir dos anos 1950 (Dezalay \& Garth, 2002). Ao analisar o papel da Fundação Rockfeller no financiamento de um esforço organizado para consolidar um campo disciplinar próprio para pensar as Relações Internacionais - que tem na Conferência sobre Política Internacional de maio de 1954 seu marco fundamental -, Guilhot e seus colaboradores vão mostrar, por sua vez, como as Relações Internacionais, em particular na sua vertente "realista", se consolidaram como uma disciplina norte-americana, que contribui para dinâmicas de poder complexas entre elites profissionais voltadas à gestão do Estado, grupos de interesse e classes sociais (Guilhot, 2011).

Essa nova historiografia crítica que tem se fortalecido recentemente, antes de enfraquecer a disciplina de Relações Internacionais, pode contribuir, na verdade, para um importante giro reflexivo que possibilite aos seus praticantes pensar criticamente seus fundamentos teóricos, metodológicos, históricos einstitucionais para, a partir disso, conquistar capacidade analítica, objetividade e autonomia. É dentro desse movimento que se deve entender a referida ampliação do debate das Relações Internacionais com outras disciplinas das Ciências Sociais. Nessa chave, esses diálogos aparecem como uma tentativa de recuperar e complexificar os fundamentos teórico-metodológicos da disciplina. Embora esse movimento de diálogo não seja propriamente novo, como o demonstra tanto a teoria crítica quanto o construtivismo em Relações Internacionais nos anos 1980, ele claramente ganha nova densidade contemporaneamente, como sugerem os trabalhos que citamos acima e outros que aparecem como referências nos textos do presente dossiê.

Esse movimento contemporâneo do campo das Relações Internacionais, ligado tanto ao fortalecimento da historiografia crítica das Relações Internacionais quanto aos novos diálogos teóricos que ela potencializa, se confronta com dois outros desdobramentos.

Por um lado, tem-se o já mencionado desenvolvimento da subárea de Economia Política Internacional (EPI) que, pelo seu caráter interdisciplinar, sempre foi mais aberta ao diálogo com outras perspectivas teórico-metodológicas e menos seduzida pela necessidade de afirmação do monopólio disciplinar sobre a temática 
do internacional (SImÉAnT, 2015). Mas a história da gênese da Economia Política Internacional também está sujeita a embates. A visão mais tradicional e consolidada atribui o surgimento da área às mudanças do sistema internacional nos anos 1970, a partir do colapso do acordo de Bretton Woods, que organizava o sistema internacional desde o final da Segunda Guerra Mundial, em particular no âmbito financeiro. Essas mudanças na institucionalidade política do sistema internacional seriam inseparáveis das transformações econômicas concomitantes, descritas mais tarde como a emergência do neoliberalismo ou, simplesmente, como o advento da globalização que reconfiguram as relações de poder em âmbito internacional. O reconhecimento da interdependência estrutural entre economia e política, que passa a marcar o desenvolvimento do sistema internacional a partir dos anos 1970, seria assim o pressuposto teórico essencial da EPI tanto na sua vertente inglesa quanto norte-americana, não obstante suas diferenças internas (BLYTH, 2009; CoHen, 2007; 2008).

Essa narrativa específica sobre o surgimento da EPI tem sido, no entanto, objeto de críticas por parte daqueles que reconhecem a área como tributária, na verdade, de estudos sobre o desenvolvimento do capitalismo na periferia, como a Comissão Econômica para a América Latina e o Caribe (CEPAL) e a teoria da dependência, na América Latina, e os estudos sobre imperialismo e colonização na África e na Ásia. Para esses autores, a ideia de que a EPI surge nos anos 1970 a partir do estudo da crise de Bretton Woods é, na verdade, bastante eurocêntrica. Seja como for, a área de Economia Política Internacional tem se institucionalizado recentemente, ganhando novas revistas, novos programas de pós-graduação e novas associações científicas como a Sociedade de Economia Política Latino-americana (SEPLA), criada no México em 2005; a International Initiative for the Promotion of Political Economy (IIPPE) do Reino Unido, criada em 2006; e a World Association for Political Economy (WAPE), sediada na China, também de 2006.

Por outro lado, esse aprofundamento da internacionalização econômica e a intensificação dos fluxos internacionais em âmbito cultural, demográfico e político, também obrigaram as demais disciplinas da Ciências Sociais a incorporar a dimensão internacional no âmbito dos seus fenômenos e teorias, o que gerou o desenvolvimento de subcampos específicos de estudo, como, por exemplo, a política internacional e comparada, a antropologia das migrações, a economia internacional e, particularmente, a sociologia das Relações Internacionais. Uma busca simples na plataforma Scielo por artigos publicados no país usando "internacional” como palavra-chave ilustra esse desenvolvimento. Considerando apenas a sociologia, por exemplo, veremos que, em 2001, apenas 9 artigos traziam "internacional” como 
palavra-chave, enquanto que em 2015, 40 trabalhos seguiam essa classificação. A mesma tendência se observa em outras disciplinas como a ciência política, a antropologia e a economia.

É nesse contexto de profusão de abordagens disciplinares e interdisciplinares sobre o internacional e suas diferentes dimensões que emergem os debates teóricos e metodológicos que o presente dossiê pretende abordar. De fato, os três primeiros textos que compõem o presente dossiê têm, como objetivo, apresentar cada uma dessas três abordagens principais: as Relações Internacionais, sobretudo na sua tradição clássica; a Economia Política Internacional, na sua vertente latino-americana; e a Sociologia das Relações Internacionais, uma área que começa a ganhar densidade no Brasil e em outros contextos nacionais.

O primeiro desses textos, $O$ conceito de Estado para os estudos realistas das Relações Internacionais: uma análise sobre a obra "A política entre as nações" de Hans Morgenthau, de Tatiana Berringer, procura analisar, justamente, a forma como a tradição realista, até hoje uma das correntes teóricas estruturantes do campo das Relações Internacionais, pensa o conceito de Estado e, mais especificamente, de Estado-nação, unidade de análise essencial para a disciplina de Relações Internacionais como um todo. Reconhecendo, justamente, que a teoria de Relações Internacionais deixou um dos seus conceitos essenciais - o conceito de Estado - “impensado", Berringer vai reconstruir como um dos teóricos fundadores da Escola Realista, Hans Morgenthau, pensava não só o Estado, mas também a nação, recuperando suas influências essenciais e mostrando como, desde a sua origem, a disciplina de Relações Internacionais está em diálogo com outras ciências sociais, em particular, no caso, a Teoria Política Clássica de Maquiavel a Weber.

No texto seguinte, intitulado A construção da nação na América Latina, um processo interrompido: bloqueios internos e externos, Carlos Serrano Ferreira e Wilson Vieira reconstroem, através de uma análise do processo interrompido de afirmação das nações latino-americanas, esquemas conceituais absolutamente essenciais para a área de Economia Política Internacional, em que o tema da nação é inseparável do desafio do desenvolvimento. Os autores recorrem ao pensamento latino-americano, em particular à teoria da dependência, para sistematizar hipóteses sobre os bloqueios internos e externos à construção nacional na região. Ao fazer isso, os autores conseguem explicitar alguns pressupostos essenciais do pensamento latino-americano em Economia Política Internacional. É importante frisar que eles acabam se voltando também para o problema do Estado mas, agora, pensado de uma perspectiva bastante distinta, segundo a qual o problema da sobe- 
rania nacional é menos geopolítico e mais econômico-social. Esvanece-se, assim, o binômio segurança-defesa e ganha destaque o problema do desenvolvimento da dependência econômica como inseparável da dominação política.

Por fim, o artigo Elementos para uma sociologia das Relações Internacionais, de Leandro Targa, traz uma tentativa de síntese teórica do que seria uma sociologia do internacional. Inspirando-se numa leitura bourdieusiana, Targa vai sistematizar, justamente, os pressupostos que mais afastam a Sociologia das Relações Internacionais de uma abordagem de Relações Internacionais stricto sensu e de Economia Política Internacional: a concepção de Estado. Não por acaso, a sociologia do Estado de Bourdieu, que vem ganhando densidade desde a publicação póstuma do seu curso sobre o Estado no Collège de France (Bourdieu, 2012) é a referência principal de Targa. Para a perspectiva sociológica sistematizada pelo autor, o Estado, como gênese de categorias essenciais de apreensão e classificação do mundo, permanece como o grande "impensado" nos principais trabalhos que tematizam o nacional e, por conseguinte, o internacional. Em suma, ao não pensar criticamente o Estado, esses trabalhos acabam "aplicando ao Estado um pensamento de Estado" (Bourdieu, 2012, p. 13).

Vistos em conjunto, portanto, esses três artigos permitem ao leitor contrastar três perspectivas disciplinares distintas sobre o conceito estruturante do campo de estudos de Relações Internacionais, a saber: o Estado e, em particular, o Estado nacional. Os artigos evidenciam como as Relações Internacionais, a Economia Política Internacional e a Sociologia definem o Estado, que problemas cada uma dessas matrizes é capaz de formular nesse bojo, o estilo de abordagem que elas desenvolvem, o tipo de linguagem e estrutura conceitual que elas projetam e o seu potencial analítico específico.

Ainda no âmbito dos artigos inéditos, o dossiê traz outras duas contribuições sobre temas bastante atuais. A primeira delas é um artigo sobre a ascensão asiática, particularmente chinesa, e o seu impacto sobre a América Latina. A segunda, uma reconstrução histórico-analítica de um dos eventos mais impactantes dos últimos anos em Relações Internacionais, o chamado Brexit, ou seja, a decisão do Reino Unido de sair da mais profunda experiência de integração regional e construção de poder supranacional vigente, a União Europeia. Apesar de tratarem de temas completamente distintos, os dois textos estabelecem um diálogo muito interessante sobre um problema essencial para qualquer ciência social: a concepção de história.

Em A importância da América Latina para a recentralização asiática do sistema-mundo, Bernardo Salgado Rodrigues e Carlos Eduardo Martins mobilizam a análise histórica estrutural, em uma perspectiva de longa duração, para 
desnaturalizar alguns pressupostos da visão histórica ocidental sobre o sistema internacional, a economia mundial e a nossa própria noção de "mundo". O artigo torna-se mais elucidativo quando lido à luz da resenha, que integra o presente dossiê, sobre o quarto volume de $O$ sistema mundo moderno e o liberalismo-centrista, de Immanuel Wallerstein. Nesse volume, Wallerstein vai analisar, de uma perspectiva macrossociológica, a história intelectual do pensamento "ocidental" que estrutura todo o debate que estamos tentando endereçar neste dossiê.

Já no texto Brexit na perspectiva do Path-dependency, Giorgio Romano também desenvolve um argumento histórico, mas a partir de uma concepção quase que oposta: ao invés de buscar as estruturas históricas que se revelam na longa duração, o autor vai tentar recuperar os eventos determinantes que mudam o curso das trajetórias históricas no médio e curto prazos. Numa tentativa de explicar o Brexit, o autor dialoga com as análises hegemônicas que interpretam esse evento apenas como um efeito conjuntural da crise de 2008, e que o entendiam como o prenúncio do desmonte da União Europeia, a partir da saída de outros países. Seu objetivo é mostrar que o Brexit se torna, na verdade, mais inteligível à luz da história do engajamento ambíguo do Reino Unido com o processo de integração europeu desde a sua origem, nos anos 1950, o que torna a decisão mais um capítulo da excepcionalidade britânica, fazendo com que seja menos provável a sua replicação.

O dossiê traz, ainda, a tradução de um artigo de Bastian van Apeldoorn intitulado Estratégia geopolítica e hegemonia de classe: para uma análise materialista-histórica de política externa. Como o título sugere, trata-se da apresentação do que seria uma perspectiva marxista das Relações Internacionais. Publicar o texto em separado faz sentido justamente porque o marxismo é a única perspectiva teórica que atravessa, com uma presença importante, as três áreas do presente dossiê: a disciplina de Relações Internacionais, a subárea de Economia Política Internacional e a Sociologia.

Este dossiê não pretende mapear todo o debate teórico-metodológico existente, hoje, no campo de estudos das Relações Internacionais. Também não pretende mapear as rápidas transformações que têm afetado esse campo, tanto na sua dimensão teórica quanto institucional. Ele pretende, antes, iniciar uma reflexão sobre esses temas, chamando a atenção para a importância de ler, reflexivamente, as disputas teóricas e metodológicas que cercam, hoje, as Relações Internacionais como objeto e como fronteira das ciências sociais. 


\section{REFERÊNCIAS BIBLIOGRÁFICAS}

ABRI. "Posicionamento da diretoria da ABRI sobre o Projeto de Lei No 8777/2017". Belo

Horizonte: Associação Brasileira de Relações Internacionais, 29 de novembro de 2017. Disponível em: http://www.abri.org.br/.

AdLER-Nissen, Rebecca. Bourdieu in International Relations: Rethinking Key Concepts in International Relations. Londres: Routledge, 2013.

Albert, Mathias \& HilkeRMeIER, Lena (orgs.). Observing International Relations: Niklas Luhmann and World Politics. Londres: Routledge, 2003.

Bigo, Didier. Pierre Bourdieu and International Relations: Power of Practices, Practices of Power. International Political Sociology, v. 5, p. 225-258, 2011.

. "Security, IR and Anthropology: Encounters, Misunderstanding and Possible

Collaborations". In: MARK, Catarina (ed.). The Anthropology of Security. Londres:

Palgrave, 2014, p. 189-205.

BLYTH, Mark (ed.). Routledge Handbook of International Political Economy (IPE). Londres: Routledge, 2009.

Bonditri, Philippe; Bigo, Didier; Gros, Frédéric (orgs.). Foucault and the Modern

International: Silences and Legacies for the Study of World Politics. Nova York: Palgrave Macmillan, 2017.

Bourdieu, Pierre. Sur L'État. Cours au Collège de France (1989-1992). Paris: Raisons d'agir, 2012.

CAmpBell, John L. "What Do Sociologists Bring to International Political Economy?”. In:

BLYTH, Mark (ed .). Routledge Handbook of International Political Economy (IPE).

Londres: Routledge, 2012, p. 260-273.

Cohen, Benjamin. The Transatlantic Divide: Why are American and British IPE so Different?'. Review of International Political Economy, v. 14, n. 2, p. 197-219, 2007. . International Political Economy: An Intellectual History. Princeton:

Princeton University Press, 2008.

Delazay, Yves \& Garth, Bryant. Le 'Washigton consensus'. Contribution à une sociologie de l'hegemonie du neolibéralisme”. Actes de la Recherche en Sciences Sociales, v. 121-122, p. 3-22, 1998.

Les usages nationaux d'une science 'globale': la diffusion de nouveaux paradigmes économiques comme stratégie hégémonique et enjeu domestique dans les champs nationaux de reproduction des élites d'État. Sociologie du Travail, v. 48, p. 308-29, 2006.

. La mondialization des guerres de palais. Paris: Seuil, 2002.

Foucault, Michael. L'archéologie du savoir. Paris: Gallimard, 2010. 
FourCADE, Marion. The construction of a global profession: the transnationalization of economics. American Journal of Sociology, v. 11, n. 1, p. 145-194, 2006.

Guilнoт, Nicolas (org.). The Invention of International Relations Theory. Nova York: Columbia University Press, 2011.

. The Democracy Makers: Human Rights and International Order. Nova York: Columbia University Press, 2005.

Latour, Bruno. Onus Orbis Terrarum: About a Possible Shift in the Definition of Sovereignty. Millennium: Journal of International Studies, v. 44, n. 3, p. 305-320, 2016.

Some Advantages of the Notion of "Critical Zone" for Geopolitics. Procedia: Earth and Planetary Science, p. 1-4, 2014.

Martin, Dominique; Metzger, Jean-Luc \& Pierre, Philippe. The sociology of globalization. Theoretical and methodological reflections. International Sociology, v. 21, n. 4, p. 499-521, 2006.

PIJL, Kees van der. The discipline of Western Supremacy. Londres: Pluto Press, 2014.

SimÉAnt, J. (org.). Guide de l'enquête globale en sciences sociales. Paris: CNRS Editions, 2015 .

Villa, Rafael; Tickner, Arlene; SouzA, Carolina; MÁsmela, Yamile. Comunidades de Relações Internacionais na América Latina: uma análise das tendências a partir do TRIP 2014. Revista Carta Internacional, v. 12, n.1, p. 224-256, 2017. 